clinical attempts about 15 years ago to treat human sepsis with antibodies against LPS. Several years later, antibodies against TNF- $\alpha$ were tried ${ }^{8}$. Both attempts failed in the clinical trials despite the earlier success in mice. If LPS is a major cause of sepsis in humans, this has not been confirmed in clinical trials.

Recent studies using models of experimental sepsis in rodents have suggested that substantial impairment of the innate immune system occurs early in sepsis (first 6 hours), often before the clinical signs of multi-organ failure $^{9}$. During this early period, neutrophil accumulation in various organs has already taken place, leading to tissue damage. Early in experimental sepsis, neutrophils appear to lose their ability to produce an 'oxidative burst' (that is, $\mathrm{H}_{2} \mathrm{O}_{2}$ production). This is likely caused by excessive amounts of the complement-activation product, C5a, which impairs assembly of NADPH oxidase ${ }^{2}$.

The latest strategy for treatment of septic patients involves the use of activated protein $\mathrm{C}$, which removes intravascular fibrin clots, although it also has other effects, such as preventing
NF- $\kappa \mathrm{B}$ activation in endothelial cells ${ }^{10}$. However, treatment of sepsis with activated protein $C$ may not represent the 'magic bullet' in humans, as it has not resulted in substantial reductions in mortality rates. Sepsis is still the number one cause for deaths in hospital intensive care units, with a mortality rate of approximately $50 \%$.

Effective therapy for the treatment of sepsis seems to be an elusive goal. The situation so far reflects the possibility that animal models do not accurately reflect the pathogenesis of sepsis in humans. It is also possible that targets for interception have yet to be found in patients with lethally impaired innate immunity during sepsis. Either way, the findings of Bochkov et al. stimulate considerable interest in determining precisely the pathophysiological role of oxidized phospholipids in sepsis. Clearly, more studies will be necessary to determine a possible therapeutical use of such reagents in humans.

1. Bochkov, V.N. et al. Protective role of phospholipid oxidation products in endotoxin-induced tissue damage. Nature 419, 77-81 (2002).

2. Czermack, B. et al. Protective effects of C5a blockade in sepsis. Nature Med. 5, 788-792
(1999).

3. Hessler, J.R., Morel, D.W., Lewis, L.J. \& Chisolm, G.M. Lipoprotein oxidation and lipoprotein-induced cytotoxicity. Arteriosclerosis 3, 215-222 (1983).

4. Leitinger, N. et al. Structurally similar oxidized phospholipids differentially regulate endothelial binding of monocytes and neutrophils. Proc. Natl. Acad. Sci. USA 96, 12010-12015 (1999).

5. Lloberas, N. et al. Postischemic renal oxidative stress induces inflammatory response through PAF and oxidized phospholipids. Prevention by antioxidant treatment. FASEB J. 16, 908-910 (2002).

6. Shimamoto, Y., Chen, R.L., Bollon, A., Chang, A. \& Khan, A. Monoclonal antibodies against human recombinant tumor necrosis factor: prevention of endotoxic shock. Immunol. Lett. 17 , 311-317 (1988)

7. Dunn, D.L., Bogard, W.C. Jr \& Cerra, F.B. Enhanced survival during murine gram-negative bacterial sepsis by use of a murine monoclonal antibody. Arch. Surg. 120, 50-53 (1985).

8. Vincent, J.L., Sun, Q. \& Dubois, M.J. Clinical trials of immunomodulatory therapies in severe sepsis and septic shock. Clin. Infect. Dis. 34, 1084-1093 (2002).

9. Huber-Lang, M. et al. Complement induced impairment of innate immunity during sepsis. Immunol. 6, 3223-3231 (2002).

10. Joyce, D.E., Gelbert, L., Ciaccia, A., DeHoff, B. \& Grinnell, B.W. Gene expression profile of antithrombotic protein $C$ defines new mechanisms modulating inflammation and apoptosis. J. Biol. Chem. 276, 11199-11203 (2001).

Department of Pathology

University of Michigan Medical School

Ann Arbor, Michigan, USA

Email: pward@umich.edu

\title{
Ain't that a Sidekick in the head
}

Neurons in the developing brain send out axons that must navigate a complex meshwork of cells to specific layers called laminae. Joshua Shanes and colleagues now show us how these axons reach their destination with precision. In the 6 September issue of Cell, they demonstrate that Sidekick-1 and -2 , transmembrane proteins in the immunoglobulin superfamily, guide axon terminals to specific laminae. This confocal image shows concentrated expression of Sidekick-2 (green) near the synaptic cleft, sandwiched between presynaptic terminals (red) and the postsynaptic apparatus (blue) in the chicken retina. Expression of Sidekick-1 in presynaptic cells targets axons to laminae expressing Sidekick-1. A similar pattern occurs between Sidekick-2-expressing presynaptic cells and Sidekick-2 laminae. The authors went on to demonstrate that each Sidekick binds to its own kind in vitro, and that in the retina their expression patterns do not overlap. Ectopic expression of either protein in Sidekick-negative cells redirects axon terminals to laminae expressing the same Sidekick. The data indicate that expression of Sidekicks in pre- and postsynaptic neurons directs formation of the synapse.

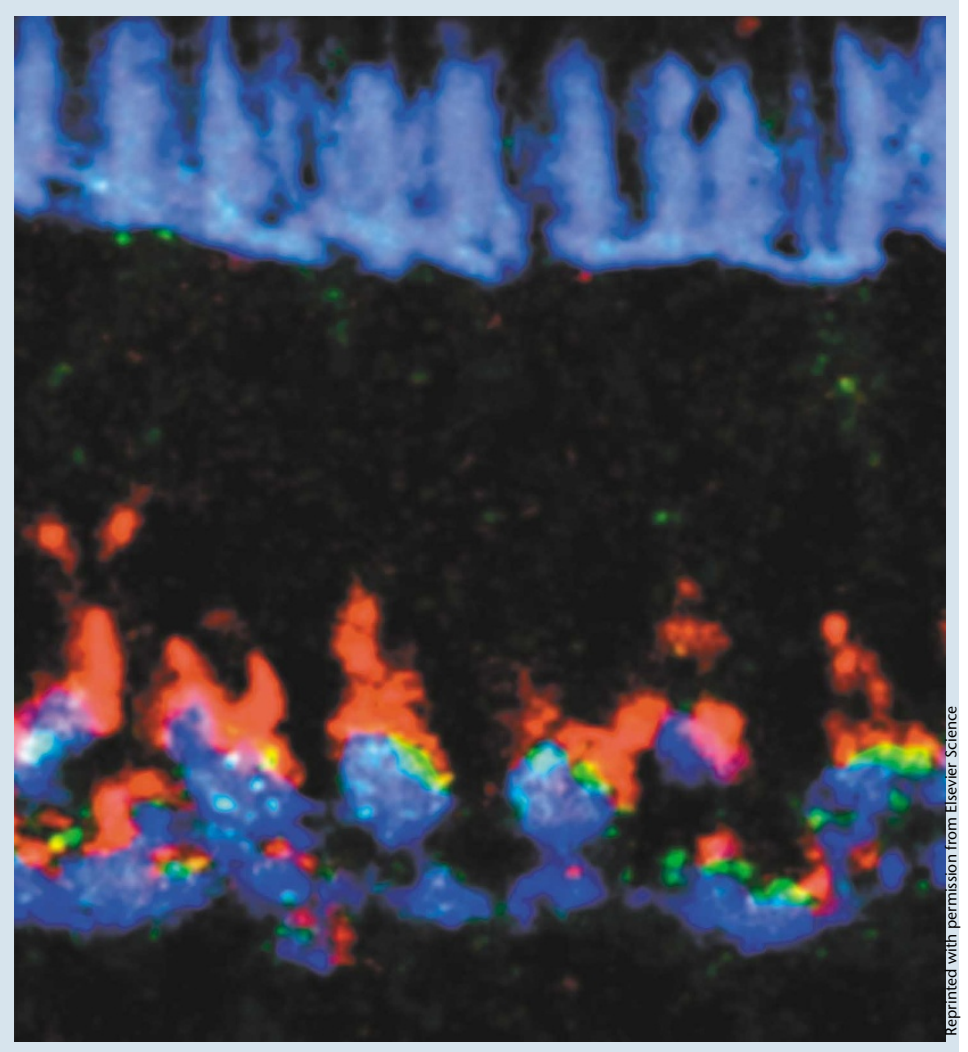

Michael Stebbins 